\title{
Regulation of a novel angiotensin II precursor, proangiotensin-12, in the tissues by blockade of the renin-angiotensin system
}

\author{
Ryousuke Satou and Hiroyuki Kobori \\ Hypertension Research (2012) 35, 153-154; doi:10.1038/hr.2011.172; published online 13 October 2011
}

$\mathrm{T}$ he renin-angiotensin system (RAS) is known to play important roles in blood pressure control and regulation of electrolytes and body fluid homeostasis. ${ }^{1}$ Previous studies have demonstrated that renin and angiotensinogen (AGT), the precursor of angiotensin (Ang) II, are expressed in multiple tissues. Therefore, a new concept of the local RAS, which functions in individual organs in a tissue-specific manner, is increasingly being recognized as an independent entity from the systemic RAS. The overactivity of the cardiac RAS has been regarded to be associated with cardiac diseases, including cardiac hypertrophy, coronary artery disease such as myocardial infarction, and hypertension., ${ }^{2,3}$ Since intrarenal Ang II is elevated in many forms of hypertension, the renal RAS is acknowledged as a key target for clinical and biochemical studies. ${ }^{4}$ In fact, overactivity of the renal RAS is considered to be associated with various kidney diseases, including glomerular sclerosis, diabetic nephropathy, and renal arterial stenosis. ${ }^{5,6}$ Furthermore, systolic blood pressure increases were accompanied by the development of renal injury by kidney-specific overproduction of Ang II in human renin/human AGT doubletransgenic mice. ${ }^{7}$ Thus, it is not doubtable that the activity of tissue RAS contributes to the development of hypertension and organ damages.

According to the classical concept, the RAS consists of renin, AGT, Ang-converting enzyme (ACE), Ang I, Ang II, and Ang II

$\overline{\mathrm{R} \text { Satou and } \mathrm{Dr} \mathrm{H} \text { Kobori are at the Departments of }}$ Medicine and Physiology, Director of the Molecular Core in Hypertension and Renal Center of Excellence, Tulane University Health Sciences Center, 1430 Tulane Avenue, \#SL39/M720, New Orleans, LA 70112-2699, USA.

E-mail: hkobori@tulane.edu type 1 and type 2 receptors, in which renin acts on AGT to produce Ang I which is processed to Ang II by ACE. Therefore, the reaction between renin and AGT was regarded as the only mechanism to produce Ang I in the classical concept of the RAS. During the past few decades, novel components of the RAS including (pro)renin receptor, ACE2, other Ang peptides and their receptors have been discovered. Findings obtained from studies of these novel RAS components have given us knowledge of novel roles of RAS in many research fields. ${ }^{8}$ In 2006, Nagata et al. ${ }^{9}$ isolated a new AGT derivative, proangiotensin-12, from rat small intestine. This peptide is abundant in the small intestine, spleen, liver and the kidney. The concentrations of proangiotensin-12 in the heart and the kidney are higher than the concentrations of Ang I and Ang II, although the concentration of proangiotensin-12 in plasma is lower than the concentrations of Ang I and Ang II. Noteworthy facts are that proangiotensin-12 is identified as a precursor of Ang II metabolized by renin-independent pathways, and that infusion of proangiotensin-12 induces vasoconstriction and high blood pressure. ${ }^{9}$ Therefore, these results provide a strong basis for the hypothesis that proangiotensin12 plays an important role to produce Ang II at the tissue levels. On the basis of this hypothesis, Nagata et al. ${ }^{10}$ investigated the contribution of proangiotensin-12 to tissue Ang II production using spontaneously hypertensive rats (SHR), and published the results in this issue of the Hypertension Research.

In this study, Nagata et al. demonstrated that both treatments with an Ang II type 1 receptor blocker (ARB) and an ACE inhibitor (ACEi) suppressed blood pressure in SHR, and these treatments increased plasma renin activity in Wistar Kyoto (WKY) rats and SHR as known phenomena. However, these RAS blockades did not affect plasma proangiotensin-12 levels in these animal models. These results indicate that the plasma RAS activity does not change the plasma proangiotensin12 levels, and that renin activity is not involved in the production of proangioten$\sin -12$ in the plasma (Figure 1). Moreover, the plasma proangiotensin-12 seems to not participate in the regulation of blood pressure because the plasma proangiotensin-12 levels were not correlated with blood pressure in the study. Since the treatment with ACEi augmented plasma Ang II levels in SHR, there was considerable concern about the inhibition of ACE activity in the model of this study. To explain this phenomenon, the authors discussed the possibility of the presence of an ACE-independent pathway for Ang II production such as a chymase-dependent pathway which can be activated by ACEi treatment.

In the cardiac left ventricle of the WKY and SHR, treatment with ACEi significantly decreased proangiotensin-12 levels. However, treatments with ARB and ACEi failed to suppress Ang I and Ang II levels in the cardiac left ventricle of the WKY and SHR, although the authors show a tendency for the RAS blockades to reduce the Ang II levels in the cardiac left ventricle of both animal models (Figure 1). In addition, Jessup et al. ${ }^{11}$ have reported that the cardiac proangiotensin-12 levels in SHR were higher than those in WKY, and the renal proangiotensin-12 levels in SHR were lower than those in WKY. In contrast, Nagata et al. ${ }^{10}$ demonstrated that the basal proangiotensin-12 levels in the cardiac left ventricle of WKY were higher than those in 


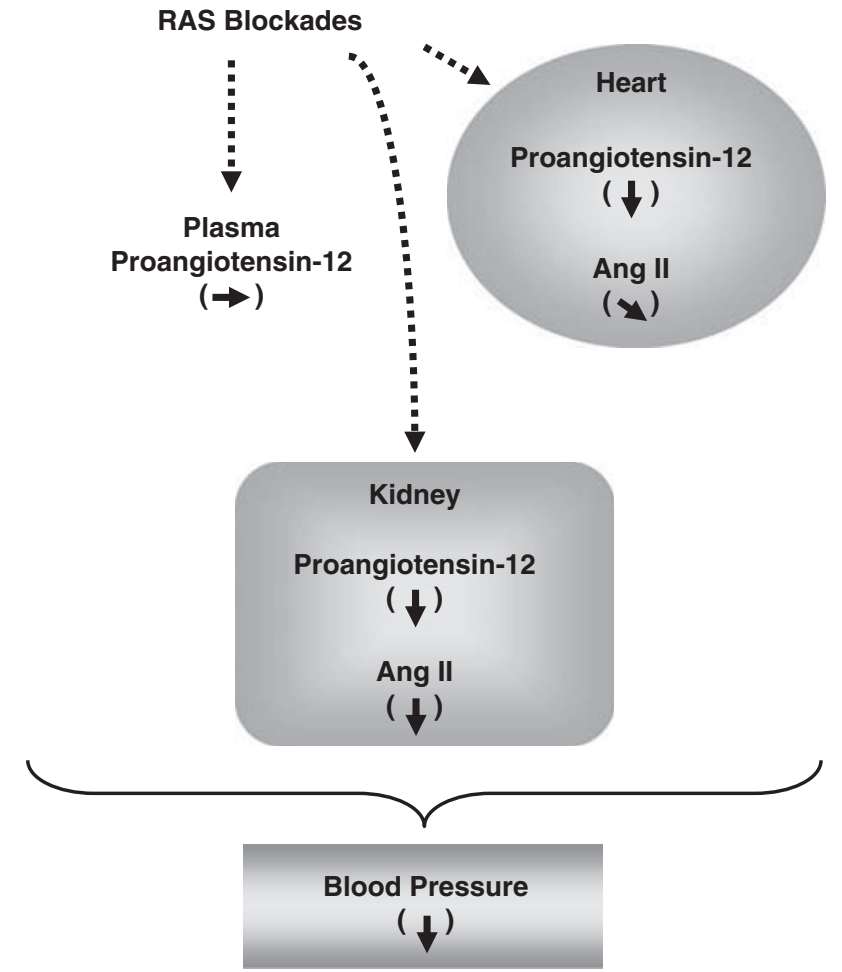

Figure 1 Proposed regulation of circulating and tissue proangiotensin-12 levels by RAS blockades. The treatments with RAS inhibitors prevent the increase in blood pressure in SHR. These RAS blockades decrease the cardiac and intrarenal proangiotensin-12 levels in WKY rats and SHR, although the treatments do not change the plasma proangiotensin-12 levels. The cardiac Ang II levels tend to be reduced by the RAS blockades, and the intrarenal Ang II levels are suppressed by the blockades.

the tissue of SHR in the study. These results suggest the possibility that the contribution of cardiac proangiotensin-12 to the production of cardiac Ang II and the development of high blood pressure is less than that in other tissues, whereas it is clear that the cardiac proangiotensin-12 levels are regulated by the RAS blockades.

In this study, the authors showed that treatments with ARB and ACEi significantly decreased the proangiotensin-12 levels in the kidneys of SHR. Furthermore, these treatments suppressed the intrarenal Ang II levels in SHR (Figure 1). Thus, these data suggest that the regulation of proangiotensin-12 levels obviously correlates with Ang II levels in the kidney, and that the intrarenal proangiotensin-12-Ang II axis plays an important role in the development of hypertension. In spite of this conclusion, the basal intrarenal proangiotensin-12 levels in SHR were lower than those in WKY in this study. To explain this controversial contradiction, it can be speculated that intrarenal proangiotensin- 12 is highly processed to Ang II in SHR; therefore, the intrarenal Ang II levels in SHR are slightly higher than those in WKY.
The mechanisms underlying the regulation of tissue proangiotensin-12 levels have not been delineated yet. In this study, the treatments with RAS inhibitors reduced the tissue proangiotensin- 12 levels. ${ }^{10}$ The authors proposed the following two possible hypotheses to explain the regulation of the tissue proangiotensin-12 levels by the RAS blockades. (1) Metabolism of proangiotensin-12 catalyzed by several proteases is enhanced by the RAS blockades, which results in reduction in the tissue proangiotensin-12 levels. This hypothesis is supported by previous reports demonstrating that proangiotensin-12 is metabolized by ACE2, chymase and neprilysin. ${ }^{9}$ To address the hypothesis, measurements of these protease activities in the tissues will be required in future studies. (2) The RAS blockades reduce the expression levels of tissue AGT, which leads to the suppression of tissue proangiotensin-12 levels. We previously reported that augmentation of intrarenal AGT expression in SHR was prevented by a treatment with an ARB..$^{12}$ Therefore, it is likely that the RAS blockades suppress the tissue proangiotensin-12 levels via the attenuation of tissue AGT augmenta- tion. Further studies will be needed to elucidate these mechanisms in hypertensive models, by evaluating correlations between the tissue proangiotensin-12 and tissue AGT levels, including urinary AGT levels.

In summary, Nagata et al. demonstrated that tissue proangiotensin-12 levels are regulated by the tissue RAS activity but not by the circulating RAS activity, which may contribute to the development of hypertension. The information obtained from this study will be valuable for further understanding the mechanisms underlying tissue RAS regulation in hypertension.

\section{CONFLICT OF INTEREST}

The authors declare no conflict of interest.

1 Navar LG, Prieto MC, Satou R, Kobori H. Intrarenal angiotensin II and its contribution to the genesis of chronic hypertension. Curr Opin Pharmacol 2011; 11: 180-186.

2 Dzau VJ, Re R. Tissue angiotensin system in cardiovascular medicine. A paradigm shift? Circulation 1994; 89: 493-498.

3 Tamura K, Tanimoto K, Takahashi S, Sagara M, Fukamizu A, Murakami K. Structure and expression of the mouse angiotensinogen gene. Jpn Heart J 1992; 33: 113-124.

4 Kobori H, Nangaku M, Navar LG, Nishiyama A. The intrarenal renin-angiotensin system: from physiology to the pathobiology of hypertension and kidney disease. Pharmacol Rev 2007; 59: 251-287.

5 Kagami S, Border WA, Miller DE, Noble NA. Angiotensin II stimulates extracellular matrix protein synthesis through induction of transforming growth factor-beta expression in rat glomerular mesangial cells. J Clin Invest 1994; 93: 2431-2437.

6 Raizada V, Skipper B, Luo W, Griffith J. Intracardiac and intrarenal renin-angiotensin systems: mechanisms of cardiovascular and renal effects. J Investig Med 2007; 55: 341-359.

7 Kobori H, Ozawa Y, Satou R, Katsurada A, Miyata K, Ohashi N, Hase N, Suzaki Y, Sigmund CD, Navar LG. Kidney-specific enhancement of ANG II stimulates endogenous intrarenal angiotensinogen in gene-targeted mice. Am J Physiol Renal Physiol 2007; 293: F938-F945.

8 Yang R, Smolders I, Dupont AG. Blood pressure and renal hemodynamic effects of angiotensin fragments. Hypertens Res 2011; 34: 674-683.

9 Nagata S, Kato J, Sasaki K, Minamino N, Eto T, Kitamura K. Isolation and identification of proangiotensin-12, a possible component of the renin-angiotensin system. Biochem Biophys Res Commun 2006; 350: 1026-1031.

10 Nagata S, Kato J, Kuwasako K, Asami M, Kitamura K. Plasma and tissue concentrations of proangiotensin-12 in rats treated with inhibitors of the renin-angiotensin system. Hypertens Res 2012; 35: 234-238.

11 Jessup JA, Trask AJ, Chappell MC, Nagata S, Kato J, Kitamura K, Ferrario CM. Localization of the novel angiotensin peptide, angiotensin-(1-12), in heart and kidney of hypertensive and normotensive rats. Am J Physiol Heart Circ Physiol 2008; 294 : H2614-H2618.

12 Kobori H, Ozawa Y, Suzaki Y, Nishiyama A. Enhanced intrarenal angiotensinogen contributes to early renal injury in spontaneously hypertensive rats. J Am Soc Nephrol 2005; 16: 2073-2080. 